\title{
Acute frailty assessment area within the emergency department: a pilot project
}

\author{
Authors: Joanne Pattinson, ${ }^{\mathrm{A}}$ Fiona Kearney, ${ }^{\mathrm{A}}$ Michael Azad, ${ }^{\mathrm{A}}$ John Gladman, ${ }^{\mathrm{A}, \mathrm{B}}$ Russell Pitchford ${ }^{\mathrm{A}}$ and Aamer $\mathrm{Ali}^{\mathrm{A}}$
}

\section{Aims}

To meet the rising demand for our acute frailty service within our emergency department (ED).

\section{Methods}

An area within our ED became available. We initiated discussions with key stakeholders within the trust and presented a business case to procure use of the space against competitors. A working group was set up involving key managers and clinicians from geriatrics, acute medicine and ED. Here we outlined staffing requirements (two nurses, one healthcare assistant, two advanced nurse practitioners, one consultant geriatrician, one specialist registrar and a physician associate and therapy team), equipment needs, working hours and patient selection (frailty) criteria. The intervention consisted of six cubicles where frail older patients could be assessed immediately by the frailty team direct from the initial assessment unit in ED using pre-defined frailty criteria. Patients were assessed by the multidisciplinary team before entering one of four pathways: discharge, admission to frailty unit, admission to the acute geriatrics, direct admission to ward. Service evaluation using daily plan-do-study-act cycles and weekly process mapping occurred to establish good practice and improvement areas. Patient admission data (time to be seen, numbers discharged and admitted, length of stay in the frailty area and number of breaches) were collected weekly over 7 weeks.

\section{Results}

Four-hundred and one patients seen by the service - vastly increased from the previous model. $131(41.07 \%)$ of patients were discharged directly from ED. Fourteen returned to the ED in 7 days (3.1\%). 69 returned with 28 days (17.0\%) - below average for our specialty as a whole. Median length of time to be seen once admitted to frailty area from IAU was 14 minutes. Usual interventions are comprehensive geriatric assessment, medication review and physical and social review.

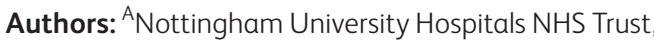
Nottingham, UK; ${ }^{B}$ University of Nottingham, Nottingham, UK

\section{Conclusion}

We were able to see many more patients than previous iterations of the service which was the key objective of the pilot. We made significant reductions in both 7 days and 28 days ED readmissions rates for our cohort of patients, reducing pressure on our hospital. We established that it is possible to have a designated frailty assessment unit that provides collaborative working across geriatrics and ED. Early assessment by the frailty team has a positive impact on readmissions of older people to ED. The area has remained operational beyond the original pilot period and we aim to continue permanently.

\section{Conflict of interest statement}

We have no conflicts of interest to declare. 